\title{
3D MODELING OF GIRIFALCO FORTRESS
}

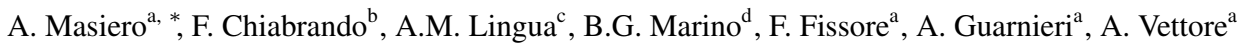 \\ ${ }^{\text {a }}$ Interdepartmental Research Center of Geomatics (CIRGEO), University of Padova, \\ Viale dell'Università 16, Legnaro (PD) 35020, Italy - (andrea.masiero, francesca.fissore, alberto.guarnieri, antonio.vettore)@unipd.it \\ ${ }^{\mathrm{b}}$ Department of Architecture and Design, Polytechnic of Turin, \\ Viale Mattioli 39, Torino, 10125, Italy - filiberto.chiabrando@ polito.it \\ ${ }^{c}$ Department of Environment, Land and Infrastructure Engineering, Polytechnic of Turin, \\ C.so Duca degli Abruzzi 24, Torino, 10129, Italy - andrea.lingua@ polito.it \\ ${ }^{\mathrm{d}}$ DiARC Department of Architecture, University of Studies Federico II, Naples Italy - bianca.marino@ unina.it
}

\section{Commission II}

KEY WORDS: Photogrammetry, UAV, Laser Scanning, Cultural Heritage Buildings, Point cloud segmentation

\begin{abstract}
:
Despite photogrammetry has been longly used for 3D model production, the recent development of structure from motion based reconstructions and the spread of unmanned aerial vehicles (UAV) are increasing its usage even further. Actually, the combined use of nadir and oblique UAV photogrammetry allows to cross the bridge between close range and aerial photogrammetry, enabling the quick acquisition of images for detailed 3D model production. This paper assesses the accuracy of UAV photogrammetry in the reconstruction of a cultural heritage building, namely the Girifalco fortress (Cortona, Italy), and it evaluates advantages and issues of this kind of 3D model generation. Furthermore, this work aims also at investigating the possible use of the generated model in order to extract geometric information about the building. First, elementary geometric shapes are extracted from the photogrammetric point cloud: the results of this step can be exploited for enabling the automatic generation of compact models of the building, which can be useful for instance in $3 \mathrm{D}$ city modeling applications. Then, a proper analysis of the geometric information included in the extracted shapes can be used in order to extract semantic information (e.g. to classify windows and doors) and to detect damaged areas on the building walls.
\end{abstract}

\section{INTRODUCTION}

The worldwide UAV market is continuously growing as also the number of applications that involve the usage of aerial drones. During the last years the use of UAVs for aerial surveys is becoming a consolidated application, commonly used for obtaining 3D models of the outer side of buildings. In accordance with this trend in outdoor building modeling, this paper considers the generation of a 3D model of the Girifalco fortress (Cortona, Italy, Fig. 11. Furthermore, in this work point cloud segmentation and classification algorithms have been applied in order to enable the automatic extraction of certain valuable information from the 3D point cloud produced by means of photogrammetric processing of images acquired by a UAV (Vosselman et al., 2004, Rabbani et al., 2006. Makuti et al., 2018).

A UAV photogrammetric survey of the Girifalco Fortress has been done by acquiring 960 images of the area of interest. Such survey, conducted on September 8, 2017, has been done by using a DJI Phantom 4, flying at an average altitude of $40 \mathrm{~m}$ over the ground (ground sample distance was $1.4 \mathrm{~cm}$, approximately). Flight plan was designed to ensure (at least) $60 \%$ of overlapping area between both successive images on the same strip and closest images from different strips. Furthermore, both nadir and oblique images have been acquired in order to improve the reconstruction accuracy of the walls of the fortress, and, concurrently, ensuring a better camera network geometry (Fraser and Stamatopoulos, 2014, Chiabrando et al., 2017).

Several widely used commercial software are available in order to obtain $3 \mathrm{D}$ reconstructions of such buildings from images acquired by UAVs (Agisoft PhotoScan, n.d. Pix4D, n.d. GonzalezAguilera et al., 2018). These tools, usually based on the Structure

\footnotetext{
${ }^{*}$ Corresponding author.
}

from Motion approach, enables 3D reconstruction with camera self calibration. Despite this can be a clear advantage in terms of saving the time required for the camera calibration, it can actually increase the reconstruction error of the obtained 3D model. The introduction of GNSS measurements in the photogrammetric reconstruction procedure, corresponding to the camera locations during image acquisitions, reduces the error level. However, ground control points should be used to ensure best performance of the photogrammetric reconstruction (Fraser, 2018).

Motivated by the above considerations, 27 targets were distributed on the area and their positions were measured by means of GNSS RTK survey (average accuracy of the topographic survey was $1.6 \mathrm{~cm})$. Ten of such targets were used as ground control points (GCPs), whereas the remaining seventeen were used as check points (CPs) for validation of the photogrammetric reconstruction, which has been performed with Agisoft PhotoScan 1.3.2.

Then, the obtained point cloud has been analyzed in order to extract information that can be useful to characterize the building geometric shape and structure, and to detect potential damages (Korumaz et al., 2017, Masiero et al., 2015). Despite commercial software is available in order to make $3 \mathrm{D}$ model geometric analysis, this paper considers the development of in-house procedures in order to make the obtained solution independent of the usage of any specific software. More precisely, the considered approach has been implemented in MathWorks Matlab, however such implementation can easily be exported in any other kind of programming language.

The obtained point cloud has been analyzed in order to detect approximately planar surfaces, which mostly corresponds to the fortress ceilings and walls. The identified surfaces can be processed further in order to obtain a synthetic simplified and com- 


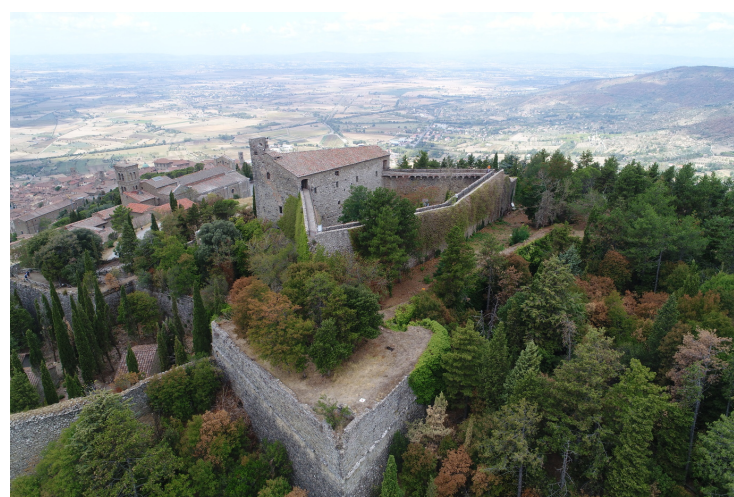

Figure 1. Girifalco fortress, Cortona, Italy.

pact model of the building (which might considered for automatically setting building geometry for producing for instance Building Information Models (BIMs) or CityGML models, (Angelini et al., 2017. Kolbe, 2009 Haala and Kada, 2010 Fissore and Pirotti, 2018, Guarnieri et al., 2017)).

Finally, a depth analysis can be used to determine windows, doors and damaged surfaces on the building walls. Actually, a classification between such cases is also investigated by using a machine learning classifier.

\section{PHOTOGRAMMETRIC RECONSTRUCTION}

DJI Phantom 4 is provided with 1 12.4 Mpix sensor, 1/2.3" CMOS The camera focal lens is $8.8 \mathrm{~mm}$, equivalent to $20 \mathrm{~mm}$ in a $35 \mathrm{~mm}$ sensor format, which ensures a field of view of $94^{\circ}$. Phantom 4 flied at an average altitude of $40 \mathrm{~m}$ over the ground, hence ground sample distance was $1.4 \mathrm{~cm}$, approximately.

Overlapping area between images along and across strips was (at least) $60 \%$. Both nadir and oblique images have been acquired in order to improve the reconstruction accuracy of the walls of the fortress, and, concurrently, ensuring a better camera network geometry.

Photogrammetric reconstruction and validation has been performed with Agisoft PhotoScan 1.3.2: a dense cloud of 40 Million points was generated with an average closest-point distance (e.g. resolution) of $1.5 \mathrm{~cm}$.

Target positions (in WGS84 UTM-ETRF2000) were obtained by a GNSS RTK survey. According to GNSS measurements, average accuracy of the topographic survey was $1.6 \mathrm{~cm}$ (Root Mean Square Error (RMSE)).

As reported in Table 1. 3D RMS error was $1.2 \mathrm{~cm}$ on GCPs, whereas it was $3.1 \mathrm{~cm}$ on CPs (planimetric RMS error was $2.0 \mathrm{~cm}$, altimetric RMS error was $2.4 \mathrm{~cm}$ ).

Table 1. Photogrammetric RMSE [cm]

\begin{tabular}{|c|c|c|c|}
\hline \hline & RMSE XY & RMSE Z & RMSE XYZ \\
\hline \hline 10 GCPs & 1.0 & 0.7 & 1.2 \\
\hline 17 CPs & 2.0 & 2.4 & 3.1 \\
\hline
\end{tabular}

Fig. 2 shows an orthophoto and a digital elevation model (DEM) of the Girifalco fortress obtained from the photogrammetric reconstruction.

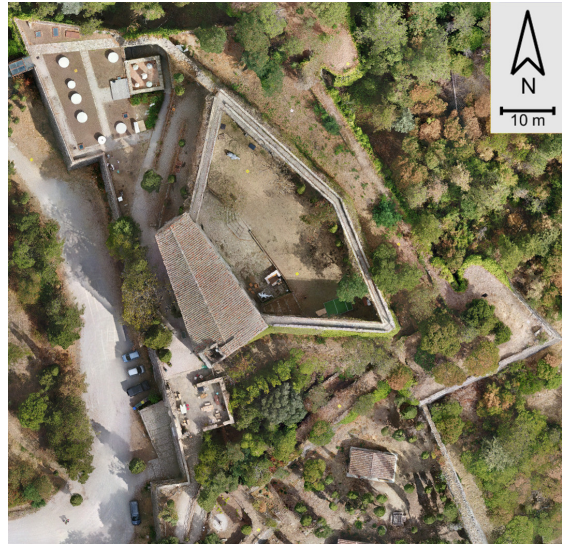

(a)

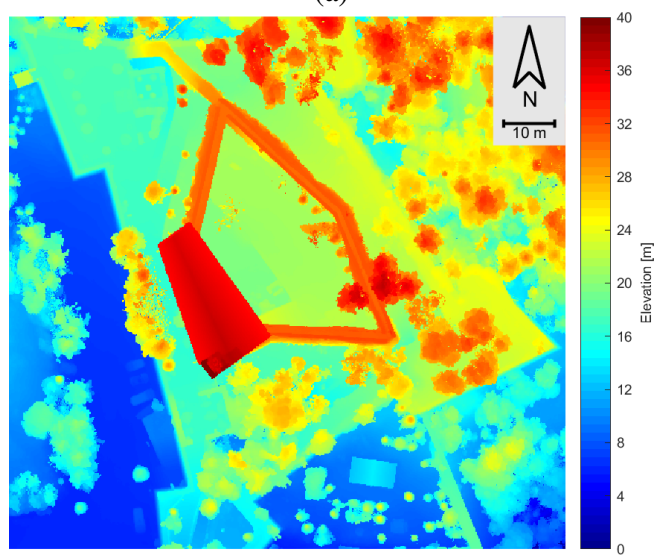

(b)

Figure 2. Orthophoto (a) and DEM (b) of the Girifalco fortress.

\section{DETECTION OF THE FORTRESS WALLS}

Point cloud obtained by Agisoft PhotoScan has been analyzed in order to automatically detect planar surfaces, which can be useful in order to produce a compact but geometrically comprehensive model of the fortress (e.g. to be used in CityGML).

To this aim, a Random Sample Consensus (RANSAC) (Fischler and Bolles, 1981) planar surface detection approach has been implemented.

First, it is worth to notice that a significant part of the surveyed area is covered by vegetation. This can lead to a negative impact on both the quality of the generated photogrammetric point cloud (in terms of both completeness and accuracy) and on the detection of planar surfaces (walls are partially, or even completely, covered by vegetation).

A local surface fitting has been used in order to check whether points approximately lies on a planar/smooth surface. The smallest eigenvalue of the sample covariance matrix of points in a local spherical neighborhood (with radius $0.25 \mathrm{~m}$ ) of the considered point has been considered in order to check the smoothed surface hypothesis (Habib and Lin, 2016), e.g. a surface locally well approximated by a plane leads to a two dimensional geometric description (i.e. small value of the third eigenvalue of the covariance matrix). This check allowed to filter out a significant part of the vegetation.

Normals to the 3D model have been computed on the local neighborhoods described above. 
Then, a RANSAC algorithm has been used to detect subsets of the original point clouds that can be well approximated with a planar surface, and whose points have associate normal directions quite similar to that of the detected plane (less than $35^{\circ}$ ).

An example of segmented planar surfaces can be seen in Fig. 3

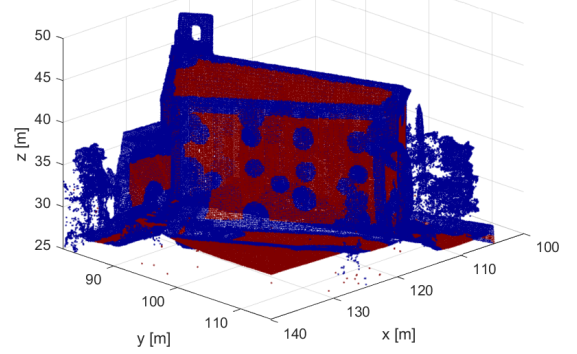

Figure 3. Segmented planar surfaces in the central part of the fortress.

Given the prior knowledge about the orientation of the obtained 3D model, the RANSAC algorithm can be tailored in order to detect vertical planar surfaces, which are those corresponding to the fortress walls, Fig. 4 Given the negative effect of the vegetation on segmentation results, walls have been delimited also by taking into account of point density and point quantity variations along each wall line.

Actually, the characteristics of the detected walls, shown in Fig. 4 can be improved further by taking into account of continuity constraints between consecutive parts of the walls. The implementation of this consideration will be considered in our future developments of this work.

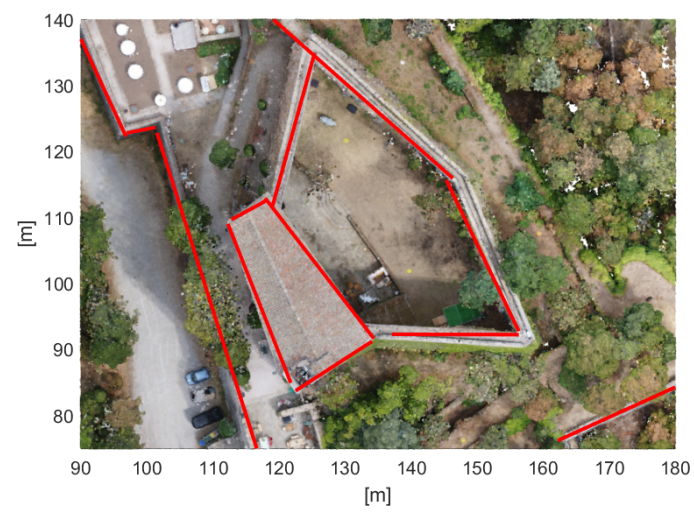

Figure 4. Detected fortress walls.

The detected wall planar surfaces can be exploited to automatically produce side/orthographic views/projections of the walls, as shown in Fig. 5

Fig. 5 clearly shows the effect of obstructions in the 3D photogrammetric reconstruction: indeed, certain areas of the walls are missing in Fig. 5. a) (bottom-left) and (b) (bottom-central part) due to the obstructions of stairs and vegetation, respectively, on images taken by the UAV.

\section{DATA ANALYSIS AND INFORMATION EXTRACTION}

Planar surface detection can be considered as a first step in order to obtain a geometric shape-based point cloud segmentation and

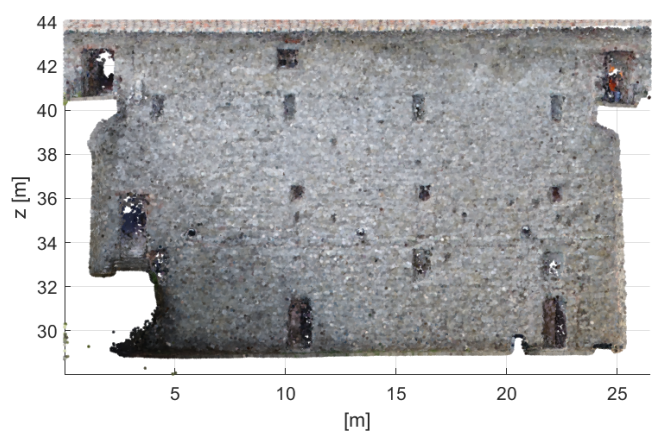

(a)

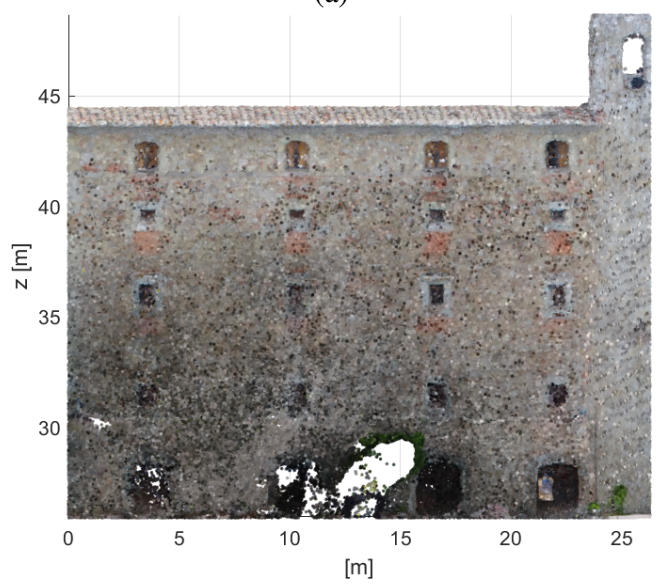

(b)

Figure 5. Side views of the central part of the fortress (a) inner wall view, (b) outer wall view.

to extract semantic information from the reconstructed model.

Motivated by the continuous need of monitoring the status of heritage buildings and by the recent spread of machine learning techniques for classification and object recognition, this Section aims at presenting certain potentialities of the recently developed machine learning techniques for automatic damage detection and recognition of architectural parts.

More specifically, geometric information extracted by the reconstructed 3D point cloud of the fortress is analyzed in order to determine areas characterized by discontinuities on the detected planar surfaces (Fig.6 a)). Then, such areas are classified in order to determine whether they are damaged areas or regular architectural parts.

It is worth to notice that a number of neural network-based techniques have recently been introduced to enhance automatic recognition abilities. Most of the currently best performing neural networks techniques are based on the use of deep learning techniques, where the network is trained by means of a typically large learning dataset. Despite the great potential of this kind of techniques, it is worth to notice that the appearance of architectural elements (e.g. doors and windows) in this case study is quite different from that of similar elements in modern buildings (e.g. Fig. 6(b)). This makes training images of this kind of elements relatively difficult to be found. This kind of techniques can actually be easily used to recognize the top row of windows in Fig. 5.b) (whose appearance is more similar to that of modern windows than the other ones in the fortress), whereas a different technique is used to recognize the other architectural elements, as described in the following. 


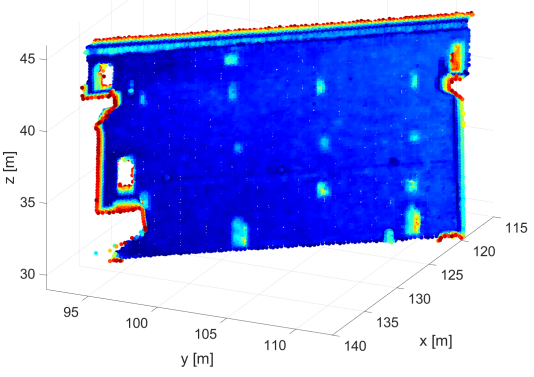

(a)

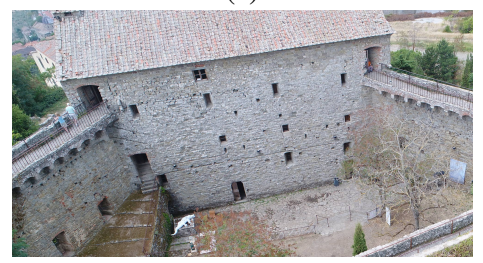

(b)

Figure 6. (a) Depth analysis on the wall of the building shown in Fig. 3 a). (b) Area corresponding to (a) shown in a UAV image.

Each wall has been analyzed searching for areas outside of the fitting plane, e.g. Fig. 7((a) has been obtained by using a proper threshold on the point-to-plane distance, which should be set depending on the photogrammetric reconstruction resolution and error).

Each connected region (with area larger than $200 \mathrm{~cm}^{2}$ ) in Fig. 7 a) is considered as a different area to be classified. For each area the following geometric information has been extracted: height, width, area.

Then, three classes were considered for classification purposes: doors, windows, and damaged parts. A decision tree has been properly trained for the classification of such classes based on the geometric information extracted as described above. Results on this case study gave a $94.1 \%$ of correct classifications.

Fig. 8 shows a misclassification example (damaged area classified as window). It is quite clear that the use of just geometric information provides relatively good results, which however should be improved by the introduction of different information (e.g. on the appearance of the area).

Furthermore, a more detailed analysis on the geometric characteristics of damaged areas can be done by checking the local pointto-planar surface distance, as shown in for instance in Fig. 9

\section{DISCUSSION AND CONCLUSIONS}

This paper presented results obtained in 3D modeling of the Girifalco fortress obtained by means of UAV photogrammetry.

UAV data collection lasted for approximately one hour, which confirms one of the typical advantages in UAV photogrammetry, i.e. the possibility of surveying relatively large areas quite fast.

The 3D model (40 M points), obtained by processing the 960 images collected by a DJI Phantom 4 , has been georeferenced using 10 ground control points, and validated by means of 17 check points, with a RMS error of $3.1 \mathrm{~cm}$ (GSD was $1.2 \mathrm{~cm}$ ).

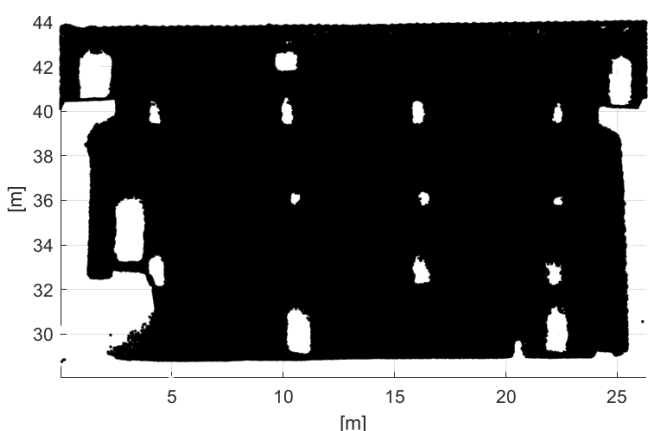

(a)

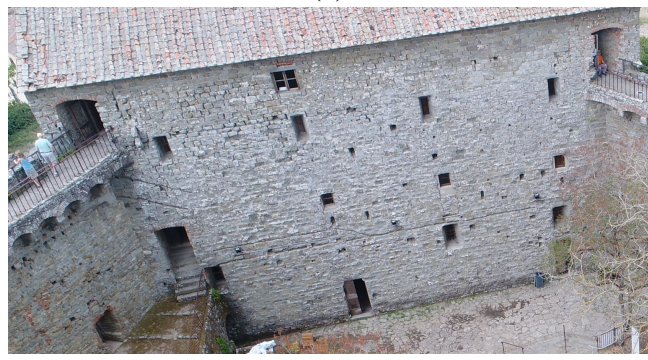

(b)

Figure 7. (a) Areas (in white) detected as not part of the inner wall of the fortress. (b) A photo of the wall analyzed in (a).

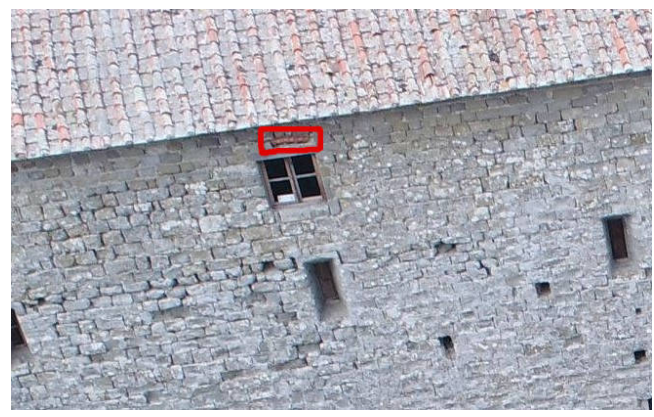

Figure 8. Misclassified area (shown within a red box).

Furthermore, standard processing of the imagery allows to obtain orthophoto and DTM of the surveyed area (He et al., 2018, Hadadin et al., 2002).

Given the obtained model accuracy, which according to the check point validation was at centimeter-level, the point cloud has been used also to automatically extract certain geometric information. First, geometric shape recognition (e.g. extraction of planar surfaces and recognition of architectural elements) has been considered: the obtained results shows the possibility of using this kind of data in order to support the production of BIMs/cityGML models (Scianna et al., 2018, Quattrini et al., 2017).

Furthermore, geometric analysis of the reconstructed walls has shown the potential use of the obtained model in order to monitor damages on the building.

Despite the aim of this paper was that of showing several potential uses of 3D models obtained by means of UAV photogrammetry, actually the obtained reconstruction allows to show also certain limits of this kind of methodology. For instance, the limitations on the flying area imposed by safety reasons (avoiding damages to the building and to the UAV) caused the impossibility of ensuring a complete coverage of the entire building by a sufficient 


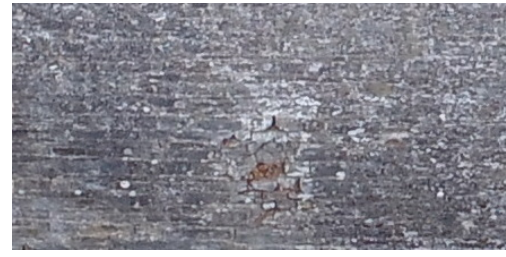

(a)

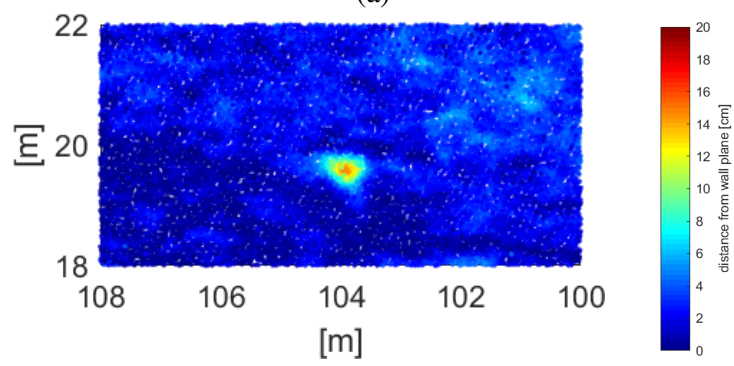

(b)

Figure 9. Close front view of a Fortress wall (a) and corresponding depth analysis with respect to the planar surface locally fitting the wall (b).

number of images. In particular, obstructions (mostly due to the presence of trees and vegetation) caused the presence of certain gaps in the model (Fig. 5), typically close to the ground. This suggests the usefulness of properly integrating UAV photogrammetry with terrestrial surveys (e.g. close-range image acquisitions (Lo Brutto et al., 2018))

Furthermore, despite the effect of vegetation has been partially reduced by properly filtering it out thanks to considerations on the local regularity of building surfaces, actually more accurate results can be obtained by using multi-spectral data acquisitions. Actually, the latter case, integrated also with LiDAR (Aicardi et al., 2016. Nex and Rinaudo, 2011. Remondino et al., 2005 Ravi et al., 2018), if possible, will be investigated in our future research work also in order to enhance the automatic recognition of objects and building architectural parts, which should be useful for instance in the production of BIMs. It is worth to notice that the considered 3D reconstruction has been obtained by exploiting highly accurate GNSS measurements: the extension of the proposed methodology to GNSS denied environments will also be considered in our future investigations (Masiero et al., 2017. Mostafa et al., 2018).

\section{REFERENCES}

Agisoft PhotoScan, n.d. http://http://www .agisoft.com/

Aicardi, I., Dabove, P., Lingua, A. M. and Piras, M., 2016. Integration between TLS and UAV photogrammetry techniques for forestry applications. iForest-Biogeosciences and Forestry 10(1), pp. 41.

Angelini, M., Baiocchi, V., Costantino, D. and Garzia, F., 2017. Scan to BIM for 3D reconstruction of the papal basilica of saint Francis in Assisi in Italy. ISPRS - International Archives of the Photogrammetry, Remote Sensing and Spatial Information Sciences XLII-5/W1, pp. 47-54.

Chiabrando, F., Lingua, A., Maschio, P. and Losè, L. T., 2017. The influence of flight planning and camera orientation in uavs photogrammetry. a test in the area of rocca san silvestro (li), tuscany. The International Archives of Photogrammetry, Remote Sensing and Spatial Information Sciences 42, pp. 163.
Fischler, M. and Bolles, R., 1981. Random sample consensus: A paradigm for model fitting with applications to image analysis and automated cartography. Communications of the ACM 24(6), pp. 381-395.

Fissore, F. and Pirotti, F., 2018. Migration of digital cartography to citygml; a web-based tool for supporting simple etl procedures. International Archives of the Photogrammetry, Remote Sensing and Spatial Information Sciences.

Fraser, C., 2018. Camera calibration considerations for UAV photogrammetry. In: ISPRS TC II Symposium: Towards Photogrammetry 2020.

Fraser, C. and Stamatopoulos, C., 2014. Automated target-free camera calibration. In: Proceedings of the ASPRS 2014 Annual Conference (Louisville, KY, USA), Vol. 2328.

Gonzalez-Aguilera, D., López-Fernández, L., RodriguezGonzalvez, P., Hernandez-Lopez, D., Guerrero, D., Remondino, F., Menna, F., Nocerino, E., Toschi, I., Ballabeni, A. et al., 2018. GRAPHOS-open-source software for photogrammetric applications. The Photogrammetric Record 33(161), pp. 11-29.

Guarnieri, A., Fissore, F., Masiero, A. and Vettore, A., 2017. From TLS survey to 3D solid modeling for documentation of built heritage: The case study of porta Savonarola in Padua. ISPRS - International Archives of Photogrammetry, Remote Sensing and Spatial Information Sciences XLII-2/W5, pp. 303-308.

Haala, N. and Kada, M., 2010. An update on automatic 3d building reconstruction. ISPRS Journal of Photogrammetry and Remote Sensing 65(6), pp. 570-580.

Habib, A. and Lin, Y.-J., 2016. Multi-class simultaneous adaptive segmentation and quality control of point cloud data. Remote Sensing 8(2), pp. 104.

Hadadin, N., Qaqish, M., Akawwi, E. and Bdour, A., 2002. Quality assessment of dtm and orthophoto generated by airborne laser scanning system using automated digital photogrammetry.

He, F., Zhou, T., Xiong, W., Hasheminnasab, S. and Habib, A., 2018. Automated aerial triangulation for uav-based mapping. Remote Sensing 10(12), pp. 1952.

Kolbe, T. H., 2009. Representing and exchanging 3d city models with citygml. In: 3D geo-information sciences, Springer, pp. 1531.

Korumaz, M., Betti, M., Conti, A., Tucci, G., Bartoli, G., Bonora, V., Korumaz, A. G. and Fiorini, L., 2017. An integrated terrestrial laser scanner (tls), deviation analysis (da) and finite element (fe) approach for health assessment of historical structures. a minaret case study. Engineering Structures 153, pp. 224-238.

Lo Brutto, M., Ebolese, D. and Dardanelli, G., 2018. 3D modelling of a historical building using close-range photogrammetry and remotely piloted aircraft system (RPAS). International Archives of the Photogrammetry, Remote Sensing and Spatial Information Sciences.

Makuti, S., Nex, F. and Yang, M., 2018. Multi-temporal classification and change detection using uav images. In: 2018 ISPRS TC II Mid-term Symposium Towards Photogrammetry 2020, 4-7 June 2018, Riva del Garda, Italy, International Society for Photogrammetry and Remote Sensing (ISPRS), pp. 651-658.

Masiero, A., Fissore, F. and Vettore, A., 2017. A low cost UWB based solution for direct georeferencing UAV photogrammetry. Remote Sensing 9(5), pp. 414. 
Masiero, A., Guarnieri, A., Pirotti, F. and Vettore, A., 2015. Semi-automated detection of surface degradation on bridges based on a level set method. ISPRS - International Archives of Photogrammetry, Remote Sensing and Spatial Information Sciences 40(3), pp. 15-21.

Mostafa, M., Zahran, S., Moussa, A., El-Sheimy, N. and Sesay, A., 2018. Radar and visual odometry integrated system aided navigation for uavs in gnss denied environment. Sensors 18(9), pp. 2776.

Nex, F. and Rinaudo, F., 2011. Lidar or photogrammetry? integration is the answer. Italian Journal of Remote Sensing 43(2), pp. 107-121.

Pix4D, n.d. https://www.pix4d.com/

Quattrini, R., Pierdicca, R., Morbidoni, C. and Malinverni, E. S., 2017. Conservation-oriented HBIM. the BIMexplorer web tool. International Archives of the Photogrammetry, Remote Sensing and Spatial Information Sciences.

Rabbani, T., Van Den Heuvel, F. and Vosselmann, G., 2006. Segmentation of point clouds using smoothness constraint. ISPRS -
International Archives of the Photogrammetry, Remote Sensing and Spatial Information Sciences 36(5), pp. 248-253.

Ravi, R., Lin, Y.-J., Elbahnasawy, M., Shamseldin, T. and Habib, A., 2018. Simultaneous system calibration of a multi-lidar multicamera mobile mapping platform. IEEE Journal of Selected Topics in Applied Earth Observations and Remote Sensing 11(5), pp. 1694-1714.

Remondino, F., Guarnieri, A. and Vettore, A., 2005. 3D modeling of close-range objects: photogrammetry or laser scanning? In: Electronic Imaging 2005, International Society for Optics and Photonics, pp. 216-225.

Scianna, A., Gaglio, G. F. and La Guardia, M., 2018. Bim modelling of ancient buildings. In: Euro-Mediterranean Conference, Springer, pp. 344-355.

Vosselman, G., Gorte, B. G., Sithole, G. and Rabbani, T., 2004 Recognising structure in laser scanner point clouds. ISPRS - International archives of photogrammetry, remote sensing and spatial information sciences 46(8), pp. 33-38. 